\title{
The compensation for movement-produced changes of object orientation*
}

\author{
HANS WALLACH, LINDA STANTON, and DEAN BECKER \\ Swarthmore College, Swarthmore, Pennsylvania 19081
}

\begin{abstract}
When one moves forward, one views objects to the side of one's path successively from different directions. In the mover's visual field, such objects change their orientation; relative to him they undergo a partial rotation. Although this rotation is given in several ways, it is hardly ever perceived. This is due to a compensating process that takes $O$ 's change in position relative to the object into account. We demonstrated the existence of this compensating process and measured the accuracy with which it operates by means of a device that made an object turn in response to $O$ 's position change so that the normal rotation of a stationary object relative to the moving $O$ could be augmented or diminished to various degrees.
\end{abstract}

It is a well-known fact that the projection of a solid object in rotation is in most cases perceived as a three-dimensional shape, often in good agreement with the shape of the object that causes the projection. Wallach and O'Connell (1953) investigated the conditions under which this happens. They also pointed out that this so-called kinetic depth effect plays a role in ordinary life, namely, when we move forward and pass a solid object. Under these conditions, the object is seen successively from different directions just as if the object were turning. Hence, a retinal projection is produced that, together with changes in size, undergoes the same form changes that the projection of the same object would undergo if the object were in partial rotation. The deformations of the retinal image that give rise to the kinetic depth effect are, therefore, the same whether 0 moves past a stationary object or whether a stationary 0 sees an object in partial rotation. No matter how these deformations are produced, the same three-dimensional object should be perceived. In another respect, however, the two conditions produce different results: In the case where the object is stationary and $\mathrm{O}$ moves, the object is not perceived to rotate. This happens in spite of the fact that the same conditions of stimulation, if produced by object rotation, would give rise to perceived rotation. Moreover, the perceived rotation that would result would not merely be the product of the kinetic depth effect. Since moving past an object results in seeing it successively from different directions and since this amounts to a rotation of the object relative to the eyes, all sensory processes that mediate the perception of rotation are in operation, in particular the processes of binocular depth perception. The present paper is concerned with the reasons why, in the end, we are not aware of this rotation.

The problem is analogous to that which is raised by

*This work was supported by Grant $25,000 \mathrm{X}$ from the National Science Foundation to Swarthmore College, Hans Wallach, principal investigator. the perceived immobility of the visual field when the head is rotated. Turning the head to the right causes the visual field to become displaced to the left relative to the head, and this displacement is identical to one caused by the visual field revolving about the stationary head. Although the objective revolving of the field would be perceived as such, the relative field displacement caused by head turning and providing identical visual stimulation conditions is not. It has been shown that this is due to a very accurate compensating process in which the neural effects of the visual displacement are matched up with proprioception of the head rotation (e.g., Wallach \& Kravitz, 1968).

There appear to be two ways to account for the nonrotation of the objects we pass. (1) Since these objects do not really turn, they maintain an unchanging orientation in relation to objects that surround them, and since we perceive the visual surround usually as stationary and rigid when we move at moderate speeds, individual objects will appear stationary also. This is the context explanation. For many years, the senior author believed it to be correct. (2) The apparent unchanging orientation of an object we pass is the result of a compensating process that matches up information about O's changing location relative to the object with the object's visually given rotation and causes its apparent immobility.

Inasmuch as the context explanation is perfectly plausible, an explanation that postulates a compensating process seems unnecessarily complex. Yet there are simple observations that argue for the operation of such a compensating process. When one walks past a realistically rendered oil portrait, the head appears to turn as if to keep looking at the person that passes by. Or better yet, when one passes a large painting of a landscape with good depth, the whole landscape appears to tum, with the foreground apparently moving with the onlooker. This is because a real head or a real three-dimensional scene would rotate relative to the moving $\mathrm{O}$ and would be perceived at rest, whereas the painted head or the picture landscape does not undergo 
such a rotation relative to the moving $\mathrm{O}$. For the context explanation to account for the apparent rotation in the pictures, the nonrotation of the painted head or scene would have to make itself felt in relation to three-dimensional objects surrounding the painting that do rotate relative to the moving 0 . This condition is usually not present.

According to the compensation explanation, the apparent rotation in pictures results from a matching up of information about $O$ 's change in location with the visually given rotation. When the latter is the normal one, that is, produced by a stationary object, no rotation is seen. But if the rotation is abnormal, a rotation of the object would be perceived. If, for any reason, the object's rotation relative to the moving $O$ were less than normal, a rotation of the object should be seen in the direction with $O$ 's progress, i.e., clockwise when the object is on $O$ 's right. The nonrotation of a picture content is an instance of such abnormal rotation with $O$ 's progress; a rotation in the direction with $O$ 's progress should be perceived.

A somewhat more unusual condition provides an even stronger argument. When one suspends a translucent plastic mask at eye level and observes it monocularly from the hollow side, it has a strong tendency to invert and look convex. When that has happened and one moves, the mask appears to turn with about twice the angle by which one gets displaced relative to the mask. This happens because a reversal of rotation direction accompanies an inversion of the rotating object. This reversal of rotation direction also occurs when the original rotation remains unnoticed. If an object is, e.g., to the right of O's motion path, O's displacement relative to the object brings about a counterclockwise rotation of the object relative to $O$, counterclockwise as seen from above. Inversion and the associated reversal of rotation direction will cause the object to appear to rotate clockwise relative to $O$, and since an O-produced counterclockwise rotation in some amount would have led to the object's apparent immobility, the inverted object will appear to rotate in approximately twice that amount. It is easy to show that the context explanation is insufficient. One can darken the room and illuminate the mask with a narrow beam from the side. Although the mask is now the only object visible, it will still appear to turn with the moving 0 through an angle larger than that of $O$ 's displacement.

We measured the accuracy of this compensating process with a device that is an analogue to the apparatus with which Wallach and Kravitz (1968) measured the constancy of visual direction. The object that was to be observed by the walking $S$ could be made to rotate objectively, in any amount and in either direction, dependent on S's change in position relative to the object. This was done by a device that variably coupled S's progress with the object's rotation. The object was fixed to a vertical shaft that was connected to the output shaft of a variable ratio transmission, while the change of S's position relative to the object caused the input shaft of the transmission to turn. Dependent on the setting of the transmission, the output shaft, together with the object, could either remain objectively stationary, or it could turn during O's passing so as to enhance the normal rotation of a stationary object relative to $\mathrm{O}$ in various proportions, or, by turning in the other sense, it could diminish that normal rotation in some proportion chosen by $\mathrm{E}$. How much of such an enhancement or diminishment would be needed for $\mathrm{O}$ to perceive the object in rotation? Or, conversely, how wide a range of enhancing or diminishing of the normal rotation relative to $O$ of a passed stationary object would go undetected? The width of such a range of undetected objective rotation would represent a measurement of the inaccuracy with which the compensation for movement produced changes of object orientation. If, e.g., the object is on the right of O's path, it will, if objectively stationary, rotate counterclockwise relative to $O$, in the amount of $O$ 's angular displacement in relation to it; this is the object's normal rotation. An objective counterclockwise rotation of the object, caused by passing on to the input shaft of the transmission O's angular displacement relative to the object, will enhance this normal rotation, and an objective clockwise rotation will diminish it. The setting of the transmission will determine the ratio of the object rotation to O's angular displacement relative to the object. This rotation/displacement ratio (RDR) will be our unit of measurement. The smaller the RDR at which the rotation of the object is perceived, the more accurate is the operation of the COO. Thus, the range of undetected object rotations, the no-rotation range for short, will be stated in terms of RDRs.

If the object is on the left of O's path, it will, if stationary, rotate clockwise relative to $\mathrm{O}$, the opposite of its rotation direction when it is on O's right side. In both cases, however, a point on the object that faces $O$ will move in the direction against O's progress, while an objective rotation in the opposite direction on either side will cause the facing surface to move objectively in the direction with 0 's forward movement. Since in our experiment $\mathrm{O}$ moves back and forth past the object and therefore has the object alternately on his right and on his left, rotation with or against 0 's progress will be the terms used for the two rotation directions. The RDR will be either in the direction with or against O's movement. Our measurements were concerned with determining the no-rotation range, the range of RDRs between the points at which object rotation in the with direction and object rotation in the against direction will be just noticed. The extent of the no-rotation range measures the accuracy with which $\mathrm{COO}$ operates.

There were two experimental conditions, one in which the object was observed in total darkness and the other in which, also in the dark, the object was seen against a background of a pattern of vertical luminous lines. The latter condition was employed to find out 
whether the object's orientation relative to a visual surround has an influence on the accuracy of the COO.

\section{EQUIPMENT}

Suspended from the ceiling was the supporting structure for four pulleys that turned on vertical shafts. The shafts were located at the corner of an oblong, $300 \mathrm{~cm}$ long in front and in the rear and $112 \mathrm{~cm}$ long on each side. Over these pulleys ran a closed loop of cable in the shape of an oblong. It was kept under tension by a fifth pulley that pulled the cable inward on one of the sides. Fastened at right angles to the cable on the front side was a $10-\mathrm{cm}$-long piece of metal tubing, into which a light hollow rod could be fitted. This rod was rigidly attached to a welder's headgear from which the glasses had been removed. When the headgear was worn, the rod rose vertically from O's forehead. When the rod was inserted into the piece of tubing, it caused the cable to move along with $O$ when he walked underneath it, sliding up and down in the tubing as O's head rose and fell with each step.

A variable ratio transmission was mounted in the center of the oblong. Attached to the vertical input shaft was a horizontal lever, a piece of metal tubing $160 \mathrm{~cm}$ long. It was inserted in a connector fixed to the cable in the rear, exactly halfway around the cable oblong from the tubing underneath which O's eyes were located. When the cable moved, the connector took the lever along and turned the input shaft of the transmission. The connector was designed to slide on rollers along the lever, as its distance from the input shaft shortened and then lengthened while it approached and passed beyond the midpoint of the $300-\mathrm{cm}$ stretch of cable. Underneath the transmission, fixed to its output shaft, was the experimental object, its height above the floor adjustable to the level of O's eyes. A handrail, $100 \mathrm{~cm}$ above the floor, ran parallel to the front cable to aid $O$ in walking underneath the cable. 1

Two different objects were used. One was a four-sided irregular wire pyramid, $6 \times 7 \mathrm{~cm}$ and painted to glow in ultraviolet light. The other object was spherical and consisted of a hollow glass bulb, $10 \mathrm{~cm}$ in diam, that was painted white and was illuminated by a small light source inside. On its surface, an irregular pattern was created with $1-\mathrm{cm}$-wide black tape. It was bright enough to be clearly visible, but did not illuminate its surround.

The pattern of vertical stripes consisted of a panel of black cardboard, $4 \mathrm{~m}$ long and $5 / \mathrm{cm}$ high, to which strips of photoluminescent tape, $1.8 \mathrm{~cm}$ wide, were attached $18 \mathrm{~cm}$ apart. This panel was illuminated by three hidden sources of ultraviolet light and was placed parallel with $O$ 's motion path $40 \mathrm{~cm}$ beyond the shaft to which the object was fastened.

\section{EXPERIMENT I}

\section{Procedure}

We measured the no-rotation range under two conditions, in total darkness or with the object seen against the striped background. The sphere was used here because we were afraid that during the lengthy measurement procedure $O$ would, in the case of the pyramid, learn to use the spatial relations between the object and the striped background in a deliberate fashion. The fact that the pyramid was a wire form whose lines could be seen crossing the stripe edges might make this possible. An abbreviated method of limits was used to measure each $O$ 's no-rotation range. In each trial, $O$ walked the length of the handrail with his head connected to the cable for its distance of about $300 \mathrm{~cm}$, stopped, turned around, and walked back to the starting point, which for half the Os was on one end of the rail and for the other half on the other end. Only during the first half of his walk to the furning point could $O$ see the sphere, for he was allowed to tum his head toward it, but not his shoulders;
Table 1

The Constancy of Object Orientation Measured With the Sphere, With and Without Stripes in its Background*

\begin{tabular}{|c|c|c|c|c|c|}
\hline \multicolumn{6}{|c|}{ Method of Evaluation } \\
\hline \multicolumn{3}{|c|}{ No Stripes } & \multicolumn{3}{|c|}{ With Stripes } \\
\hline $\begin{array}{l}\text { With } \\
\text { Limit }\end{array}$ & $\begin{array}{l}\text { Against } \\
\text { Limit }\end{array}$ & Range & $\begin{array}{l}\text { With } \\
\text { Limit }\end{array}$ & $\begin{array}{l}\text { Against } \\
\text { Limit }\end{array}$ & Range \\
\hline .402 & .240 & $\begin{array}{l}\text { No-Rotation } \\
.652\end{array}$ & $\begin{array}{c}\text { Range } \\
.359\end{array}$ & .254 & .611 \\
\hline .342 & .297 & $\begin{array}{l}\text { Uncertainty } \\
.639\end{array}$ & $\begin{array}{r}\text { Range } \\
.367\end{array}$ & .273 & .635 \\
\hline
\end{tabular}

*All means given in RDR.

the same was true of the retum walk. When the latter was completed, O reported whether the sphere had appeared to turn or not, and in which direction. A trial thus consisted of two passages. Initially, the transmission setting was such that 0 clearly saw the sphere tuming as he walked past it. On subsequent walks, the objective rotation of the sphere was diminished in steps of .04 RDR. This procedure was continued until $O$ reported no rotation on three subsequent trials. Then the transmission setting was changed to produce a clearly noticeable rotation in the opposite direction, and the other outer limit of the no-rotation range was determined in corresponding fashion. For half the Os, the procedure started with objective rotation with O's progress, and for the other half in the direction against it. After the two outer limits of the no-rotation range were obtained, the inner limits were determined with two series of trials, which, starting with transmission settings that produced immobility of the sphere, increased the rotation rate by .04 RDR steps until Os reported rotation in three subsequent trials. One series determined the inner limit in the with direction, and the other was used to find it in the against direction.

Twelve Os, mostly high school students, participated in both conditions. Half of them first made the measurements with the striped background present and a week later in total darkness. For the other six Os, the reversed order was used.

\section{Results}

Two methods of evaluating our raw data were used, and the results of both are presented in Table 1 . The first method used all limits that were obtained. The limits of the no-rotation range were computed for each $\mathrm{S}$, one toward perceived rotation in the with direction and the other in the against direction. Each of these limits was the midpoint between the first no-rotation report occurring in the run toward the no-rotation range and the first rotation report when the trial series started with no-rotation judgments. The means of these two limits and the mean no-rotation range are presented in the first row of Table 1.

When we found that the inner limits, the change from no-rotation to rotation reports, showed less variability than the outer limits obtained in the first two runs, we made another computation, using only the last two runs of the procedure, the trial series that started with no-rotation reports. Here we used as limits the first rotation report that was succeeded by two further rotation reports. These results are presented in the second row of Table 1 , headed "uncertainty range." A 
Table 2

Mean Limits and Mean No-Rotation Ranges for Pyramid and Sphere in RDR

\begin{tabular}{lccc} 
& $\begin{array}{c}\text { With } \\
\text { Limit }\end{array}$ & $\begin{array}{c}\text { Against } \\
\text { Limit }\end{array}$ & Range \\
\hline Mean & & $\begin{array}{c}\text { Pyramid }(\mathrm{N}=19) \\
.439\end{array}$ & .910 \\
SD & .480 & & .379 \\
& & Sphere $(\mathrm{N}=13)$ & \\
Mean & .376 & .439 & .815 \\
SD & & & .254 \\
\hline
\end{tabular}

comparison of the four ranges presented in the table shows that the two methods of evaluation produced essentially the same results.

The important comparison is between the ranges obtained with and without stripes. They were approximately the same for the two conditions. The confidence limits at the .05 level for the two no-motion ranges were .12 and .22 . The presence of the stripes had not much, if any, effect. Whether one concludes from this result that the visual context provided by a well-lit environment does not contribute to the apparent immobility of an object one passes depends on whether one accepts the striped background as representative of a well-lit field. But, since our results leave no doubt that a $\mathrm{COO}$ operates, this is not an important issue. Moreover, it has no bearing on other discussions of our results.

\section{EXPERIMENT II}

The purpose of this experiment was to ascertain whether the sphere used in Experiment I was a representative object where our investigation was concerned. To this end, we compared its no-rotation range with that of the pyramid. Because the pyramid is a wire shape and its near and far edges are therefore simultaneously visible, its orientation relative to $O$ is optimally given and is, in that respect, superior to any solid shape.

\section{Procedure}

The procedure of Experiment II differed from that of Experiment I only in minor ways. To shorten the procedure, $O$ reported what he had observed after each passage rather than after walking back and forth. A second change seemed necessary because of the use of the wire form: $E$ changed the orientation of the object relative to the shaft on which it turned after each passage before $O$ turned to walk in the other direction. He put it into one of four standard orientations that differed from each other by $90 \mathrm{deg}$. In the case of the pyramid, the four orientations were those where its axis formed an angle of $45 \mathrm{deg}$ with $O$ 's path. The starting orientation for each passage was randomly selected.

A different sample of Os, drawn from our undergraduate population, was used in the two experimental conditions. Measurements for the sphere were taken with 13 Os and for the pyramid with 19 .

\section{Results}

Mean limits and mean no-rotation ranges with their standard deviations are presented in Table 2. The mean no-rotation range for the sphere was somewhat larger than in the first experiment, but the difference was far from significant. No significant difference between the no-rotation ranges for the two objects was found. But since the mean range for the pyramid was somewhat larger than that for the sphere, it seems safe to conclude that the latter does not disfavor COO.

Individual differences were very large. The with limit for the sphere, which averaged .376, varied from .121 to .628 . The no-rotation range, with a mean of .815 , ranged from .344 to 1.49 .

\section{DISCUSSION}

The $\mathrm{COO}$ emerges from our measurements as a rather crude function. But it is accurate enough to account for the observation we want to explain. When one moves past a painting of a three-dimensional object, the object does not turn relative to $O$ and is not given with an optical rotation. According to our explanation, the object is perceived to rotate because its normal optical rotation is absent. If the painted object were real, it would have to turn at 1.0 RDR in the with direction in order to produce this optical nonrotation. This is well beyond the mean limits of the no-rotation ranges we have measured and beyond any limit in the with direction we have encountered in individual Ss.

The question arises, what use is a compensating function with a no-rotation range of .8 or .9 RDR? Such a no-rotation range means that objective rotations that occur during $O$ 's locomotion and fall within that no-rotation range will not be perceived, although most of them could undoubtedly be perceived were $O$ stationary. To be sure, we are not seriously disadvantaged by such an arrangement. But, then, why does $\mathrm{COO}$ exist at all when it yields perception of existing rotation during locomotion only outside such a large no-rotation range? A simpler arrangement by which all optical rotations that occur during one's locomotion are prevented from causing perceived rotation would be as useful.

There is little hope that this question can be answered by further investigation of $\mathrm{COO}$. To be sure, $\mathrm{COO}$ is very likely the result of an adaptation acquired some time in $O$ 's past; it should therefore be possible to modify it through experimental adaptation, as has been done in the case of the constancy of visual direction. But such an approach does not appear feasible. One of the reasons why experimental adaptation in the constancy of visual direction can be so easily investigated is its very small no-motion range, which makes partial adaptation easily measured (Wallach \& Frey, 1969; Wallach, Frey, \& Romney, 1969; Wallach \& Floor, 1970). The very large no-motion range of $\mathrm{COO}$ makes this impossible.

The answer to the question may eventually be found in connection with the investigation of a class of adaptation processes that resemble each other. In all of them, the perceptual effects of those exteroceptive 
stimuli which are caused by O's own movements are eliminated. Such sensory inputs do not carry useful information, i.e., information about genuine environmental events. They are easily detected by the nervous system because they always occur when the movements that cause them take place. They are therefore covariant with certain kinesthetic processes, namely, the proprioceptions of those movements. Once a connection between these correlated exteroceptive and proprioceptive inputs has been established, a compensating process can develop that eliminates the normal effect of the exteroceptive stimulation. This class of adaptation processes is thus based on a covariance principle. COO can be regarded as the result of such an adaptation developed in $O$ 's past. The exteroceptive input whose perceptual effect has been eliminated results from the optical rotation of an object past which one moves. The kinesthetic processes with which this input is covariant are either the proprioceptions of the movements that cause one to view objects from different vantage points or, more likely, the movements made necessary by one's changing location relative to the object. The latter consist of the gradual turning of one's eyes and one's head out of the direction in which one walks (that is, out of the primary position), a combination of rotary movements that take place when one moves past an observed object. The covariance between this combination of rotary movements and the object's optical rotation is not a simple linear one but of a higher order, because, for both, the rotation rate increases as one approaches the object.
The constancy of visual direction also fits this scheme. Here, the rate of displacement of the visual field caused by the head rotation varies with the rate of the head rotation. Both the original adaptation process that caused this constancy and the experimental adaptation that modifies it obviously belong to this class. We know of at least one other type of experimentally produced adaptation that develops only when the sensory events whose perceptual results are being diminished by the adaptation are covariant with O's head movements. It is interesting that this case of adaptation is not related to a normally existing compensation process (Wallach \& Barton, in preparation).

\section{REFERENCES}

Wallach, H., \& Floor, L. On the relation of adaptation to field displacement during head movements to the constancy of visual direction. Perception \& Psychophysics, 1970, 8, 95-98.

Wallach, H., \& Frey, K. J. Adaptation in the constancy of visual direction measured by a one-trial method. Perception \& Psychophysics, 1969, 5, 249-252.

Wallach, H., Frey, K. J., \& Romney, G. Adaptation to field displacement during head movement unrelated to the constancy of visual direction. Perception \& Psychophysics, $1969,5,253-256$.

Wallach, H., \& Kravitz, J. H. Adaptation in the constancy of visual direction tested by measuring the constancy of auditory direction. Perception \& Psy chophysics, 1968, 4, 299-303.

Wallach, H., \& O'Connell, D. N. The kinetic depth effect. Journal of Experimental Psychology, 1953, 45, 205-217.

\section{NOTE}

1. We thank Otto Hebel for designing this apparatus.

(Received for publication July 11, 1973; revision received November 5,1973 .) 